\title{
ON MONIN-OBUKHOV SIMILARITY IN THE STABLE ATMOSPHERIC BOUNDARY LAYER
}

\author{
MARKUS PAHLOW ${ }^{1,2}$, MARC B. PARLANGE ${ }^{1,2}$ and FERNANDO PORTÉ-AGEL ${ }^{3}$ \\ ${ }^{1}$ Department of Geography and Environmental Engineering, ${ }^{2}$ Center for Environmental and \\ Applied Fluid Mechanics, The Johns Hopkins University, Baltimore, MD 21218, U.S.A.; \\ ${ }^{3}$ Department of Civil Engineering, University of Minnesota, Minneapolis, MN 55414, U.S.A.
}

(Received in final form 17 August 2000)

\begin{abstract}
Atmospheric measurements from several field experiments have been combined to develop a better understanding of the turbulence structure of the stable atmospheric boundary layer. Fast response wind velocity and temperature data have been recorded using 3-dimensional sonic anemometers, placed at several heights $(\approx 1 \mathrm{~m}$ to $4.3 \mathrm{~m})$ above the ground. The measurements were used to calculate the standard deviations of the three components of the wind velocity, temperature, turbulent kinetic energy (TKE) dissipation and temperature variance dissipation. These data were normalized and plotted according to Monin-Obukhov similarity theory. The non-dimensional turbulence statistics have been computed, in part, to investigate the general applicability of the concept of $z$-less stratification for stable conditions. From the analysis of a data set covering almost five orders of magnitude in the stability parameter $\zeta=z / L$ (from near-neutral to very stable atmospheric stability), it was found that this concept does not hold in general. It was only for the non-dimensional standard deviation of temperature and the average dissipation rate of turbulent kinetic energy that $z$ less behaviour has been found. The other variables studied here (non-dimensional standard deviations of $u, v$, and $w$ velocity components and dissipation of temperature variance) did not follow the concept of $z$-less stratification for the very stable atmospheric boundary layer. An imbalance between production and dissipation of TKE was found for the near-neutral limit approached from the stable regime, which matches with previous results for near-neutral stability approached from the unstable regime.
\end{abstract}

Keywords: Atmospheric turbulence, Monin-Obukhov similarity, Stable atmospheric boundary layer.

\section{Introduction}

Monin-Obukhov similarity (Monin and Obukhov, 1954) for the atmospheric boundary layer (ABL) under unstable stability has been extensively studied (see Businger et al., 1971; Wyngaard et al., 1971; Kaimal et al., 1976; Kader and Yaglom, 1990). There is a long history of field experiments contributing to the continuing development of Monin-Obukhov similarity (e.g., Takeuchi 1961; Businger et al., 1971; Wyngaard and Coté, 1971; Merry and Panofsky, 1976; Nieuwstadt, 1984a; Sorbjan, 1986; Brutsaert, 1992, Parlange and Katul, 1995; Mahrt et al., 1998). The experimental results for unstable conditions are extensive and are routinely used in applications (Parlange et al., 1995). Considerably fewer studies exist that cover the very stable boundary layer, even though this is of great 
practical importance (Mahrt, 1998). Some insight into the structure of the stably stratified ABL has been gained through several studies (e.g., Caughey et al., 1979; Nieuwstadt, 1984a,b; Smedman, 1988) and a number of more recent experimental investigations on Monin-Obukhov similarity under stable stability have been published (e.g., Dias et al., 1995; Dias and Brutsaert, 1996; Forrer and Rotach, 1997; Howell and Sun, 1999; Mahrt, 1999). Similarity under stable stratification remains less well studied. Progress in understanding boundary-layer turbulence under stable stability is made more slowly than for the unstable case due to its sensitivity to drainage forces generated by even slight terrain slopes (Caughey et al., 1979), low-level jets, the influence of gravity waves (Finnigan, 1999) and other mesoscale motions (e.g., cold air drainage and meandering motions) (Mahrt, 1998). Knowledge of the turbulence structure under stable atmospheric conditions remains of great importance, especially for applications in air pollution. Other areas that will greatly benefit from an improved description of the stable atmospheric boundary layer include flux-dissipation methods for heat and momentum calculations (Parlange et al., 1999) and numerical modelling of the boundary layer with large-scale models or large-eddy simulation (LES) models.

In this work, extensive data sets from several field experiments are combined to study Monin-Obukhov similarity under stable stratification. The stability range considered here extends to strong atmospheric stability relative to most other field observations. Under strong stability, Monin-Obukhov similarity theory suggests that all turbulence statistics become independent of the height $z$ above the ground. In this study we examine that suggestion, sometimes referred to as $z$-less stratification (Wyngaard and Coté, 1972).

\section{Background}

\subsection{Monin-OBUKhov SIMILARITY}

Monin-Obukhov (hereafter M-O) similarity theory for the ABL was developed for a stationary atmospheric surface layer over horizontally homogeneous terrain. The structure of turbulence is determined by the kinematic surface stress $\tau_{0} / \rho$, the buoyancy parameter $g /\langle\theta\rangle$, the surface heat flux $\left\langle w^{\prime} \theta^{\prime}\right\rangle_{0}$, and the height above ground $z$, where $\tau_{0}$ is the surface shear stress, $\rho$ is the air density, $g$ is acceleration due to gravity, $w^{\prime}$ is the vertical velocity fluctuation, $\theta^{\prime}$ is the temperature fluctuation and $\langle\cdot\rangle$ denotes the averaging operation. These parameters are needed to define the velocity, temperature and length scales. Generally the friction velocity $u_{*}$, the temperature scale $\theta_{*}$, height above ground $z$ and the Obukhov length $L$ are chosen as surface-layer scales. They are defined as follows:

$$
u_{*}=\left(\tau_{0} / \rho\right)^{1 / 2}=\left(-\left\langle u^{\prime} w^{\prime}\right\rangle_{0}\right)^{1 / 2}
$$




$$
\begin{aligned}
& \theta_{*}=\frac{-\left\langle w^{\prime} \theta^{\prime}\right\rangle_{0}}{u_{*}}, \\
& L=\frac{-u_{*}^{3}\langle\theta\rangle}{k g\left\langle w^{\prime} \theta^{\prime}\right\rangle_{0}},
\end{aligned}
$$

where $u^{\prime}$ is the longitudinal velocity fluctuation and $k(=0.4)$ is von Karman's constant.

Dimensional analysis shows that an atmospheric turbulence property or statistics, such as gradients, variances and covariances, when properly scaled, are universal functions of the stability parameter $\zeta(=z / L)$ (Brutsaert, 1999). The exact forms of these functions are not predicted by theory and have to be determined through field experiments.

It is often useful to consider the limits of surface-layer similarity under extreme unstable (so-called 'free convective') or extreme stable (' $z$-less') stability conditions. Here we focus on the case of very stable stratification. For this limit it has been suggested that $z$ is no longer of importance (Monin and Yaglom, 1971) and it was apparently first termed ' $z$-less stratification' by Wyngaard and Coté (1972). Under strong stable stratification, the existence of large-scale turbulent fluctuations becomes impossible (since these fluctuations would have to expend too much energy in performing work against the gravity forces), and turbulence can exist only in the form of small scale eddies (Monin and Yaglom, 1971, p. 437). If stable stratification increases even further, the eddy size should be limited entirely by stability and not by the distance from the surface. The three remaining parameters are the kinematic surface stress $\tau_{0} / \rho$, the buoyancy parameter $g /\langle\theta\rangle$ and the surface heat flux $\left\langle w^{\prime} \theta^{\prime}\right\rangle_{0}$.

\subsection{BUdGET EQUATIONS FOR TURBULENT KINETIC ENERGY AND TEMPERATURE VARIANCE}

The time averaged turbulent kinetic energy budget equation per unit mass for stationary flow over a homogeneous surface is

$$
-\left\langle u^{\prime} w^{\prime}\right\rangle \frac{\partial U}{\partial z}+\frac{g\left\langle w^{\prime} \theta^{\prime}\right\rangle}{\langle\theta\rangle}-\frac{\partial\left\langle e w^{\prime}\right\rangle}{\partial z}-\frac{1}{\rho} \frac{\partial\left\langle p^{\prime} w^{\prime}\right\rangle}{\partial z}=\epsilon,
$$

where $U$ is the mean longitudinal velocity, $e=(1 / 2)\left(u^{\prime^{2}}+v^{\prime^{2}}+w^{\prime^{2}}\right)$ is the TKE, $p^{\prime}$ is the fluctuating component of pressure and $\epsilon$ is the mean dissipation rate of TKE (Stull, 1988). The first two terms on the left hand side represent the production rates of turbulence by shear and buoyancy, respectively. The third term on the left is the divergence of vertical turbulent flux and the fourth term describes the transport due to pressure. The term on the right hand side is the viscous dissipation term. Equation (4) is non-dimensionalized by multiplying (4) with $k z / u_{*}^{3}$ :

$$
\phi_{m}-\frac{z}{L}-\phi_{t}-\phi_{p}=\phi_{\epsilon} \text {. }
$$


Numerous studies have been carried out to find an expression for the dimensionless shear (Dyer and Hicks, 1970; Businger et al., 1971; Högström, 1988; Beljaars and Holtslag, 1991; Howell and Sun, 1999). A linear relation for the shear production of the form $\phi_{m}=1+\beta(z / L)$ is usually used to fit observations over the entire stable range. This form also satisfies the concept of $z$-less scaling for the limit of very strong stability (Wyngaard and Coté, 1972). A universal value for the constant $\beta$ has yet to be found under stable conditions. For the dimensionless dissipation $\phi_{\epsilon}$ Wyngaard and Coté (1971) suggested $\phi_{\epsilon}=\left[1+2.5(z / L)^{3 / 5}\right]^{3 / 2}$. Another form, $\phi_{\epsilon}=1+5(z / L)$ was given in Kaimal and Finnigan (1994) assuming the normalized dissipation rate to be unity for neutral conditions. It is often argued that, for neutral stability, buoyant destruction $(z / L)$ and the transport terms $\left(\phi_{t}\right.$ and $\left.\phi_{p}\right)$ are equal to zero and therefore shear production equals dissipation. It has been found in a number of studies that this is not the case (Högström, 1990a; Frenzen and Vogel, 1992; Albertson et al., 1997).

In the stable surface layer the contribution of the individual terms in (5) is often assumed to be $\phi_{m} \simeq \phi_{\epsilon}$ and $\phi_{t} \simeq 0$, and therefore transport due to pressure has to balance buoyant destruction. But as Kaimal and Finnigan (1994) point out, the question of energy balance in the stable layer remains unresolved. In particular, uncertainty exists for the very stable stratified boundary layer and in the present work we address some of these unresolved issues.

For steady, horizontally homogeneous flow the budget equation for temperature variance (actually the budget for $\theta^{\prime 2} / 2$ to be consistent with the form of the TKE) is

$$
-\left\langle w^{\prime} \theta^{\prime}\right\rangle \frac{\partial \Theta}{\partial z}-\frac{1}{2} \frac{\partial\left\langle w^{\prime} \theta^{\prime 2}\right\rangle}{\partial z}=\epsilon_{\theta}
$$

where the first term is the average production rate $(\Theta$ is the mean air temperature), the second term represents the flux divergence of the temperature variance and $\epsilon_{\theta}$ is the mean dissipation of the temperature variance (Stull, 1988). Equation (6) can be non-dimensionalized by $k z u_{*} /\left\langle w^{\prime} \theta^{\prime}\right\rangle^{2}$ to obtain

$$
\phi_{h}-\phi_{T}=\phi_{\epsilon_{\theta}} \text {. }
$$

Functional relationships for the dimensionless production of temperature variance $\phi_{h}$ have been proposed. A commonly used form is $\phi_{h}=1+\gamma(z / L)$. No universal value for the constant $\gamma$ has been found yet. Often, the transport term is found to be negligible compared to $\phi_{h}$ and $\phi_{\epsilon_{\theta}}$ (Wyngaard and Coté, 1972; Champagne et al., 1977; Panofsky and Dutton, 1984). This leads to an even more simplified budget for the temperature variance: $\phi_{h}-\phi_{\epsilon_{\theta}}=0$. We will focus in our study on the dissipation of temperature variance. 


\subsection{Methods to DETERMine MEAN Dissipation of TKE, $\epsilon$ AND TEMPERATURE VARIANCE, $\epsilon_{\theta}$}

In order to evaluate the mean dissipation of turbulent kinetic energy $\epsilon$, direct or indirect methods can be used. Direct methods require measurements that extend to very small scales, preferably to the Kolmogorov microscale $\eta(\sim 1 \mathrm{~mm}$ in the $\mathrm{ABL}$ ) to obtain meaningful results. In this study we will resort to the so-called indirect methods, and will use second- and third-order structure functions to obtain $\epsilon$ and $\epsilon_{\theta}$.

The second- and third-order longitudinal structure functions $\left(D_{u и}\right.$ and $\left.D_{u u u}\right)$ and the second-order temperature and mixed velocity-temperature structure functions ( $D_{\theta \theta}$ and $D_{u \theta \theta}$ ) of locally isotropic turbulence are related through

$$
\begin{aligned}
& D_{\text {иии }}(r)-6 v \frac{d D_{u и}(r)}{d r}=-\frac{4}{5}\langle\epsilon\rangle r, \\
& D_{u \theta \theta}(r)-2 \chi \frac{d D_{\theta \theta}(r)}{d r}=-\frac{4}{3}\left\langle\epsilon_{\theta}\right\rangle r,
\end{aligned}
$$

where $v$ is the kinematic viscosity of air, $\chi$ is the molecular diffusivity for heat, $\epsilon$ is the turbulent kinetic energy dissipation rate per unit mass, $\epsilon_{\theta}$ is the dissipation rate of the temperature variance and $r$ is the separation distance in the longitudinal direction (Yaglom, 1949; Monin and Yaglom, 1975, pp. 395-401). These equations are satisfied for sufficiently large Reynolds and Peclet numbers. In the surface layer, an appropriate Reynolds number is $R e=\frac{u_{*} z}{\nu} \approx O\left(10^{5}\right)$. Since the Peclet number and the Reynolds number are related by $P e=\frac{v}{\chi} R e$ the requirements for the above equations (8) and (9) to be valid are certainly met in the ABL. For the limit $r \gg \eta$ (which corresponds to the inertial subrange), viscous friction and molecular thermal conduction are negligible, which simplifies (8) and (9) to

$$
\begin{aligned}
& D_{\text {иии }}(r)=-\frac{4}{5}\langle\epsilon\rangle r, \\
& D_{u \theta \theta}(r)=-\frac{4}{3}\left\langle\epsilon_{\theta}\right\rangle r .
\end{aligned}
$$

We draw our conclusions from the results obtained with the third-order structure functions, as opposed to the second-order structure functions, since the numerical coefficients in (10) and (11) are exact (von Karman and Howarth, 1938). The use of second-order structure functions requires one to choose values for the free constants involved, which would add uncertainty to the analysis.

Using these results and combining them with the definition of the third-order structure function which represents the averaged cubed velocity differences over lag $r$ (Monin and Yaglom, 1975, Ch. 8)

$$
D_{\text {иии }}(r)=\left\langle(u(x+r)-u(x))^{3}\right\rangle,
$$


the mean dissipation rate of TKE, $\epsilon$ can be calculated. Similarly, the mean dissipation rate of temperature variance, $\epsilon_{\theta}$ can be obtained. The mixed third-order structure function, representing the product of the longitudinal velocity difference and the squared temperature difference as a function of lag $r$, is given by

$$
D_{u \theta \theta}(r)=\left\langle(u(x+r)-u(x))(\theta(x+r)-\theta(x))^{2}\right\rangle .
$$

Combining (11) and (13) yields the mean dissipation rate of temperature variance.

\section{Experiments}

For all five experiments considered in this study 3-dimensional sonic anemometers have been used to measure the three velocity components $(u, v, w)$ and the air temperature $(\theta)$. The two types of sonic anemometers used for these experiments were a Gill triaxial ultrasonic anemometer (Gill Instruments/1012R2) with a pathlength $d_{s l}=0.149 \mathrm{~m}$ and a Campbell Scientific CSAT3 sonic anemometer $\left(d_{s l}=0.1\right.$ $\mathrm{m})$. The basic working principle for sonic anemometers of this type is that the air movement effect on the transit time of sound pulses travelling in opposite directions across a known instrument path length $d_{s l}$ is measured. This is, the wind velocity vector projected on the acoustic path is measured. A disadvantage of the sonic anemometer is the wavelength distortion due to line-averaging along the sonic path $d_{s l}$. According to Kaimal (1986), the distortion is confined to wavelengths smaller than $2 \pi d_{s l}\left(=\lambda_{d}\right)$, and in order to obtain undistorted spectral response, this limiting wavelength will be taken into account for the analysis.

Table I shows a summary of the experiments. The total number of runs used for this study is 479. The Davis data have been collected at the Campbell Tract research field at the University of California at Davis and the Iowa data were obtained during a field experiment in Amana, Iowa. The sites were flat fields with long fetch (between $400 \mathrm{~m}$ and $700 \mathrm{~m}$ ) in the upwind direction and with varying surface cover (bare soil, grass and beans). This will allow us to draw conclusions from the results without the possible bias by data collected at one site with a specific surface cover.

This extensive data compilation for a wide range of stable atmospheric conditions (more than five orders of magnitude in the stability parameter $z / L$ ) provides more insight into the behaviour of atmospheric turbulence for the stable case. But one has to be aware of uncertainties arising from different sources and their influence on the results. Therefore great care has to be taken while performing the experiment to limit the sources of possible errors and uncertainties. For the data presented in this study, the recording time for individual runs was between 20 and 30 minutes, which guarantees a large enough sample size of the flux carrying eddies. Depending on the recording frequency, this results in samples ranging from roughly 20,000 to 100,000 data points per run. For an increasing number of data points one can expect more reliable results from the statistical analysis. On the 
TABLE I

Summary of experiments.

\begin{tabular}{llllll}
\hline & Davis 1994 & Davis 1995 & Davis 1996 & Iowa 1998 & Davis 1999 \\
\hline sensor height [m] & 1.54 & 1.07 and 0.96 & 1.7 & 1.63 to 4.14 & 1.79 to 4.32 \\
\hline Sampling frequency [Hz] & 21 & 21 & 18 & $20-60$ & 20 \\
Sampling period [min] & 20 & 20 & 20 & 30 & 30 \\
Number of runs & 48 & 130 & 41 & 53 & 207 \\
Surface cover & beans & bare soil; beans & bare soil & grass & bare soil \\
Canopy height [cm] & 13 & 0 to 15 & - & 15 & - \\
\hline
\end{tabular}

other hand, stationary atmospheric conditions are required to satisfy the general applicability of surface-layer similarity theory. Therefore a sampling period has to be chosen that is long enough to allow for reliable statistics and short enough to fulfill the stationary condition and to avoid flux sampling problems, which are often met under stable conditions (Nieuwstadt, 1984a; Mahrt, 1985; Högström, 1988; Howell and Sun, 1999). Every run has been inspected individually to prevent erroneous results arising from trends in the dataset or unsteady conditions during the run.

\section{Results}

\subsection{TURBULENCE STATISTICS}

To guarantee the suitability of the conversion from the time domain to the space domain using Taylor's (1938) frozen turbulence hypothesis the turbulence intensity ( $T I=\sigma_{u} /\langle u\rangle$, where $\sigma$ denotes the standard deviation) of each run has been computed. It has been suggested that Taylor's hypothesis should be valid when the turbulence intensity is small compared to the average wind speed (Wyngaard and Clifford, 1977; Stull, 1988; Peltier et al., 1996) and this criterion has been applied successfully in several studies (e.g., Kiely et al., 1996; Albertson et al., 1997, Katul et al., 1997; Tong et al., 1998, 1999; Porté-Agel et al., 1998, 2000, 2001). Data files with $T I>0.5$ have been rejected to satisfy the requirement for the applicability of Taylor's hypothesis. This screening reduced the number of files for the analysis from originally 479 to 446 . The coordinate system has been aligned with the mean wind for each file. The velocity scale $u_{*}$ was computed from the longitudinal stress, as defined by (1).

Figure 1 shows the normalized standard deviation of the $u$-velocity component as a function of the stability parameter $\zeta=z / L$. The data suggest that the scaled $u$-standard deviation is constant up to $\zeta \approx 0.1$. The constant value for $\sigma_{u} / u_{*}$ in 
this weakly stable region was found to be 2.3. This is in agreement with the value (=2.3) obtained by Smedman (1988), and also agrees well with the data in Hsieh and Katul (1997) (their Figure 15b). For very stable conditions, $\sigma_{u} / u_{*}$ increases strongly. This behaviour has also been observed by other researchers (Smedman, 1988; De Bruin et al., 1993; Chu et al., 1996; Hsieh and Katul, 1997). For strongly stable stratification the turbulent motion is damped ( $u_{*}$ becomes small) and for increasing $\zeta$ the stabilizing effect dominates. The turbulent eddies are no longer directly influenced by the surface and the length scale $z$ becomes unimportant. This stable limit was termed 'local $z$-less stratification' (Wyngaard and Coté, 1972). The data presented in this study show constant behaviour only for $\zeta \lesssim 0.1$, where both the velocity standard deviation $\sigma_{u}$ and the friction velocity $u_{*}$ increase with height in the same manner, and departs from being constant for stronger stability. To cover the entire stability regime with one single expression for all three velocity components, a general expression to describe the functional relationship $\sigma_{\alpha} / u_{*}=$ $f(z / L)$, where $\alpha$ represents the three velocity components, was chosen,

$$
\frac{\sigma_{\alpha}}{u_{*}}=a+b(z / L)^{c} .
$$

By performing a least squares analysis the parameters for the $u$-velocity component were found to be $a=2.3, b=4.3$ and $c=0.5$. This reduces to $\sigma_{u} / u_{*}=2.3$ in the limit $z / L=0$, as desired. The plot of (14) with these fitted coefficients is presented in Figure 1.

Figure 2 shows the normalized values for the $v$-velocity standard deviation for the same range of stability as in Figure 1. As for the $u$ component, the ratio $\sigma_{v} / u_{*}$ remains constant for $\zeta \lesssim 0.1$. This is followed by a transition regime. Beyond that the normalized $v$-standard deviation does not show $z$-less behavior but increases rapidly. The value for the constant range for weaker stability was found to be 2.0, somewhat larger than Smedman's result, which was 1.7. The least squares analysis gave the following parameters for the functional relationship (14) between the normalized $v$-velocity standard deviation and the stability parameter $z / L: a=2.0$, $b=4.0$ and $c=0.6$. Again, the constant value is retained for the neutral limit $(\zeta=0)$. The fitted curve is plotted in Figure 2.

The third turbulence property considered is the standard deviation of the vertical velocity. Whereas only few published data sets for the $u$ and $v$ components exist, results for the $w$ component can be found frequently in the literature (e.g., Merry and Panofsky, 1976; Kondo et al., 1978; Nieuwstadt, 1984a; Smedman 1988; De Bruin et al., 1993; Chu et al., 1996; Mahrt et al., 1998). Even though the scaling behaviour of the $w$ component seems to be relatively well known, uncertainties still exist under strong atmospheric stability. In Figure 3 the standard deviation of the $w$ component $\sigma_{w}$, normalized with $u_{*}$, is plotted versus $\zeta(=z / L)$. In this case, the values of $\sigma_{w} / u_{*}$ approach a constant value of 1.1 for $\zeta \lesssim 0.1$. Again, we find a transition regime and for stronger stability $\sigma_{w} / u_{*}$ begins to increase (for $\zeta>0.1$ ). The values for the universal expression (14) were obtained to be $a=1.1, b=0.9$ 


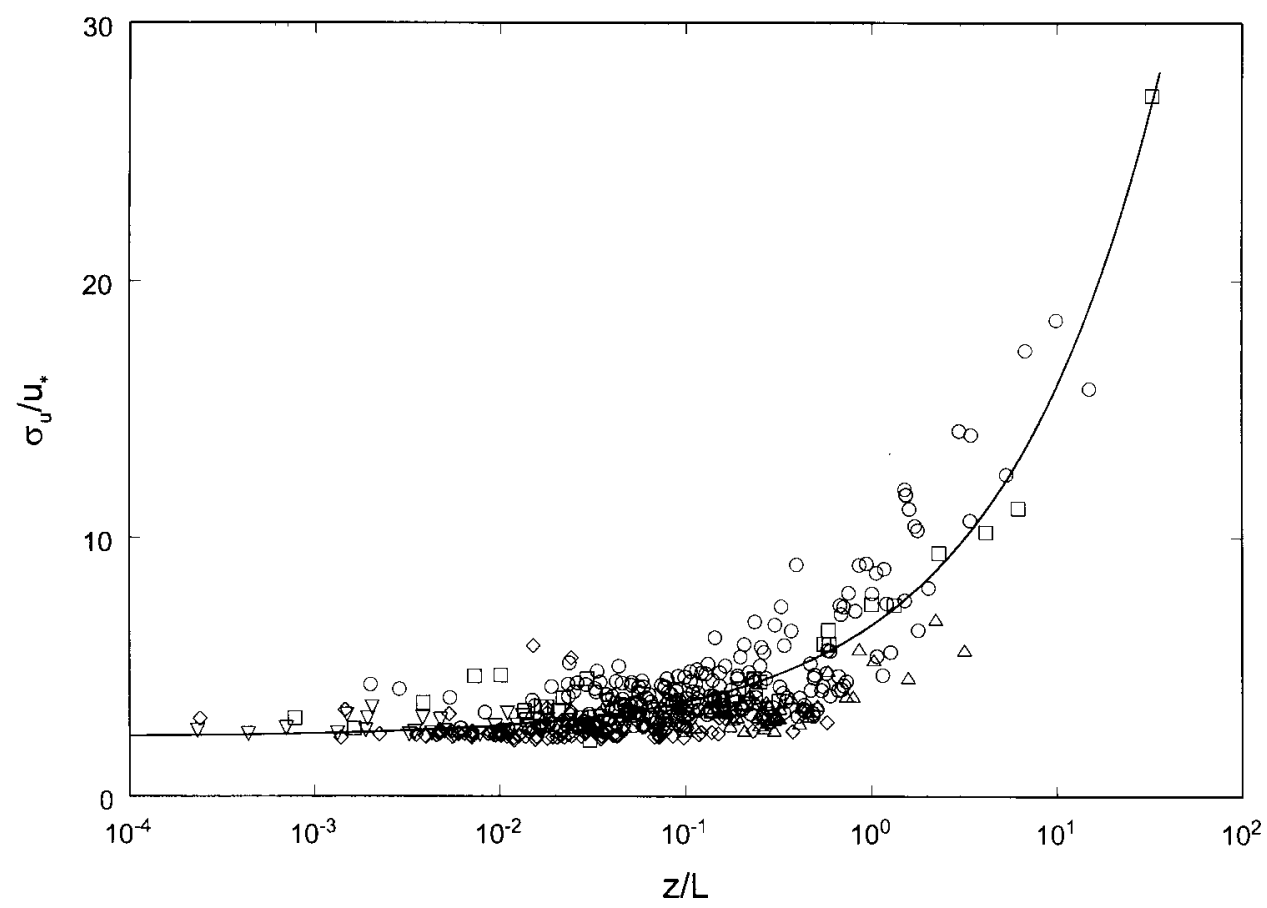

Figure 1. $u$-velocity standard deviation normalized with friction velocity $u_{*}$. Symbols represent data from field experiments in Davis 1999 (circles), Iowa 1998 (upward pointing triangles), Davis 1996 (squares), Davis 1995 (diamonds) and Davis 1994 (downward pointing triangles); solid line represents best fit.

and $c=0.6$, which is plotted with the data in Figure 3. Dias et al. (1995) give a review of the values found for the constant (termed $A_{w}$ ) under stable conditions. Upon computing an average value for those results obtained by different authors we find $A_{w}=1.32$, slightly larger than the value found here $(a=1.1)$.

The plots for the three velocity components strongly support the scaling laws obtained, in particular for two reasons: (1) The large dataset (446 runs) covers a very broad range of $\zeta$ from near neutral to very stable conditions $\left(\zeta_{\max }=32.9\right)$ with qualitatively very little scatter. (2) The data were collected over various terrain (bare soil, beans and grass). This excludes bias due to environmental conditions in the field.

Högström (1990b) suggests that the general increase of the normalized standard deviation of the velocity fluctuations is due to the contribution of non-turbulent mesoscale motions ('inactive turbulence') to the variances. This hypothesis was supported by the results obtained by Mahrt et al. (1998), in which the variables were recomputed using a 100-s averaging period instead of the original 5-min averaging period, in order to eliminate the non-turbulent motion. This resulted in a smaller $\sigma_{V} / u_{*}$ (where $V=\left[u^{\prime 2}+v^{\prime 2}\right]^{1 / 2}$ ) for large $\zeta$ values. Also, the relationship between $\sigma_{w} / u_{*}$ and $\zeta$ remained the same, suggesting that the increase in variance 


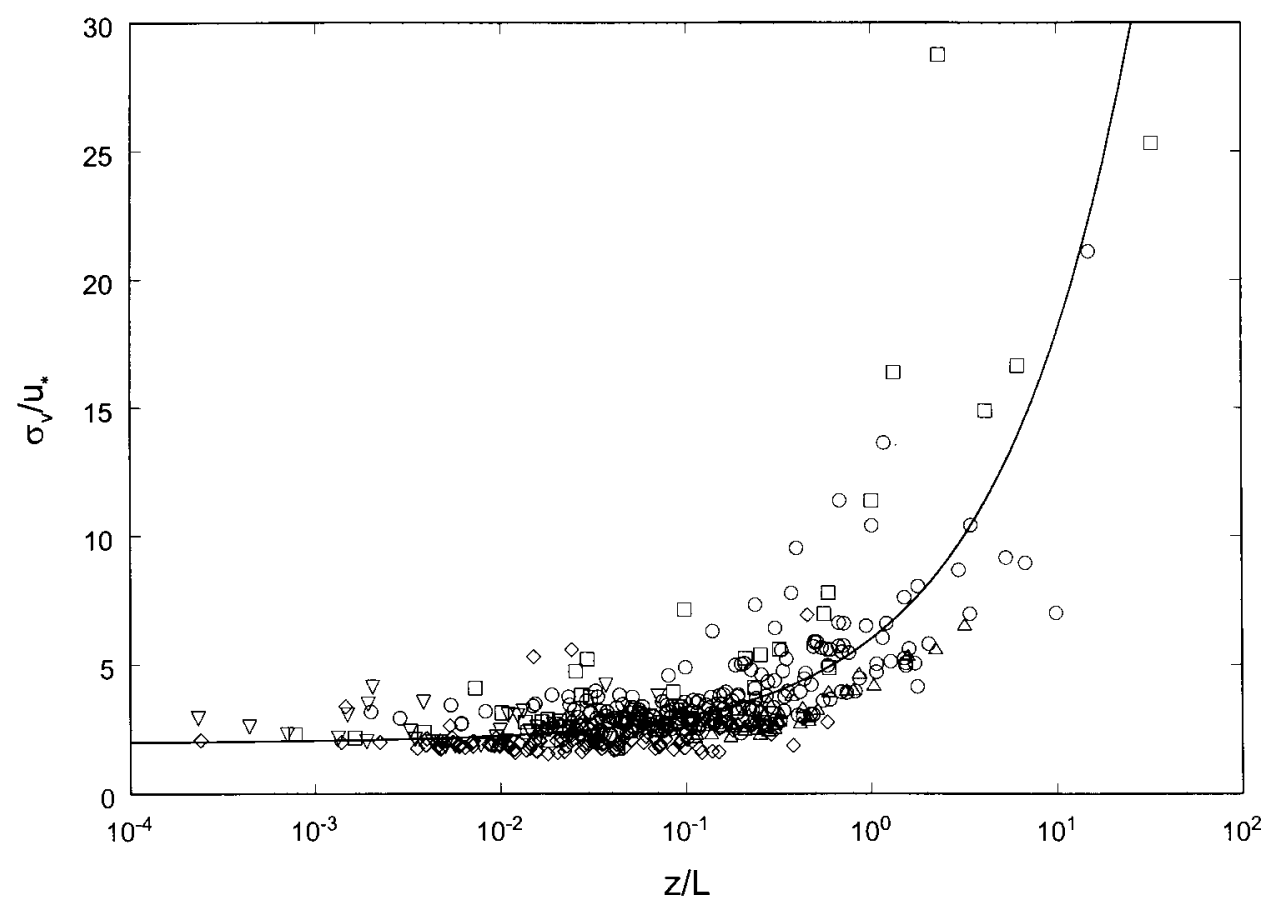

Figure 2. $v$-velocity standard deviation normalized with friction velocity $u_{*}$ (symbols are the same as in Figure 1).

is mainly due to horizontal motions. To fully accept this hypothesis, more studies are needed. It is yet not clear if the effect can be explained by horizontal motions. In the present work, the coordinate system has been aligned with the mean wind, reducing $\langle v\rangle$ to zero. However, the fluctuating components both increase for strong stable conditions.

It should be noted here, that the functional relationship $\sigma_{w} / u_{*}=f(\zeta)$ is possibly influenced by self-correlation between $\sigma_{w}$ and $u_{*}$, as was pointed out by Mahrt et al. (1998). In Figure 4 the standard deviation of the vertical velocity fluctuation is shown as a function of the friction velocity. The relationship is almost linear. Since $u_{*}$ is used to normalize the standard deviation and appears in the Obukhov length (defined in (3)), the correlation between those two parameters might be partly due to self-correlation.

We continue our discussion on surface-layer scaling under stable conditions by presenting the results for the normalized standard deviation of temperature as a function of $\zeta$ in Figure 5a. For near-neutral conditions $\sigma_{\theta} / \theta_{*}$ increases strongly, whereas it levels off for increasing $\zeta$. Since the vertical scale for $\sigma_{\theta} / \theta_{*}$ is large due to the strong increase for near-neutral conditions, the results are re-plotted on a smaller scale where the largest values have been omitted (Figure 5b). It can be seen from this figure that the scatter is larger than for the velocity fluctuations, but 


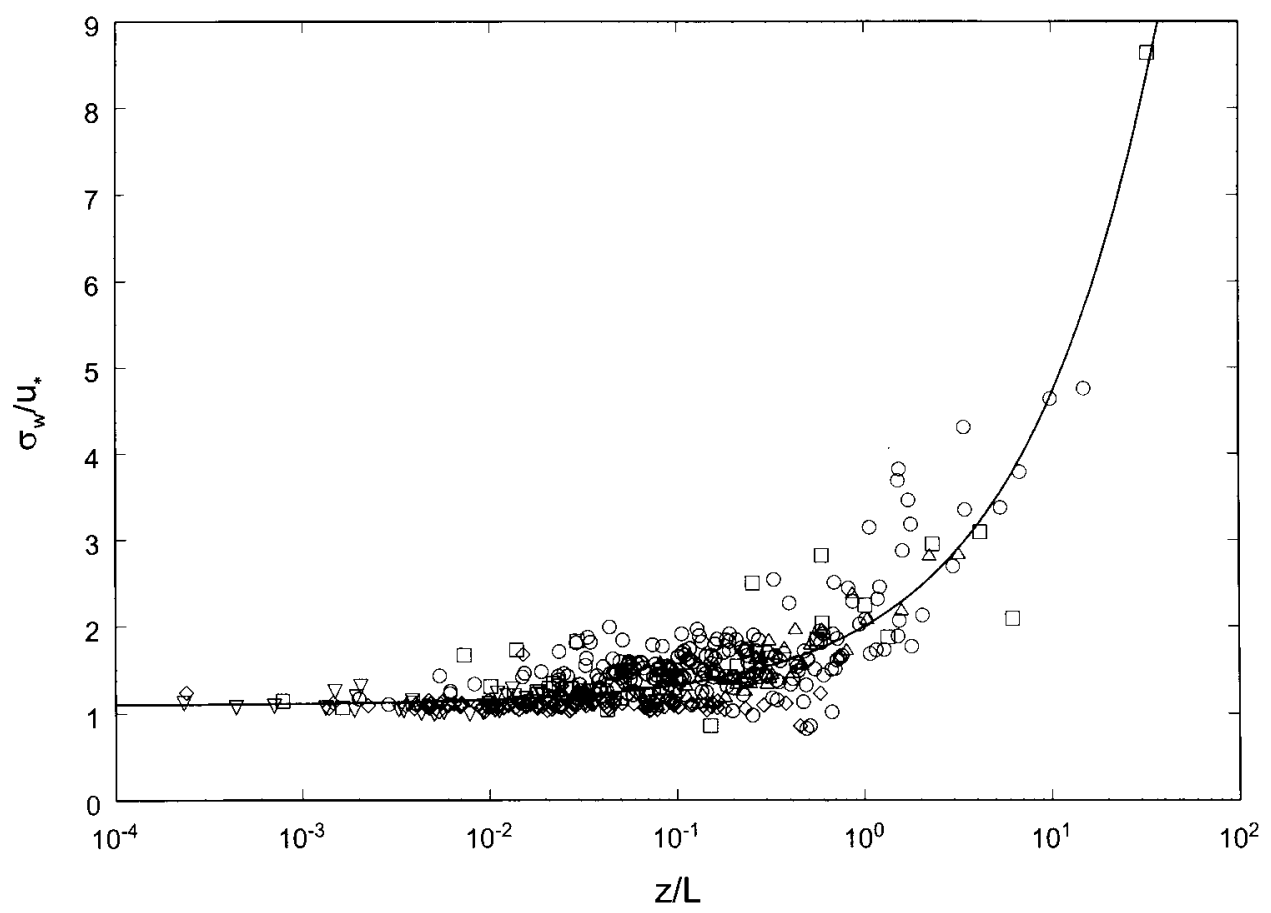

Figure 3. $w$-velocity standard deviation normalized with friction velocity $u_{*}$ (symbols are the same as in Figure 1).

$\sigma_{\theta} / \theta_{*}$ becomes independent of $z$ for increasing $\zeta$. The constant value for the normalized temperature standard deviation was found to be 3.0; this value should be viewed with caution due to the large scatter observed in the data. Other researchers found values fairly close to this result. For example, Wang and Mitsuta (1991) and Nieuwstadt (1984a) also found a value of 3.0 and De Bruin et al. (1993) found the constant to be 2.9. The large increase towards neutral conditions can be explained by considering the temperature scale, which is defined as $\theta_{*}=-\left\langle w^{\prime} \theta^{\prime}\right\rangle / u_{*}$. The magnitude of the heat flux decreases for decreasing stability, which leads to large values for $\sigma_{\theta} / \theta_{*}$ when the neutral limit is approached. An expression for the functional relationship between $\sigma_{\theta} / \theta_{*}$ and $z / L$, which includes the effect of weak heat flux for near-neutral conditions, is

$$
\frac{\sigma_{\theta}}{\theta_{*}}=a(z / L)^{b}+c,
$$

with $a=0.05, b=-1$ and $c=3$, which is plotted along with the data in Figure $5 \mathrm{a}, \mathrm{b}$.

Next, the variation of the negative sensible heat flux with stability is further studied. Figure 6 shows $\left\langle w^{\prime} \theta^{\prime}\right\rangle$ as a function of $z / L$. As was mentioned before, the heat flux decreases for near-neutral stability. For the other limit, under very stable atmospheric stratification, vertical movement is suppressed by a damping 


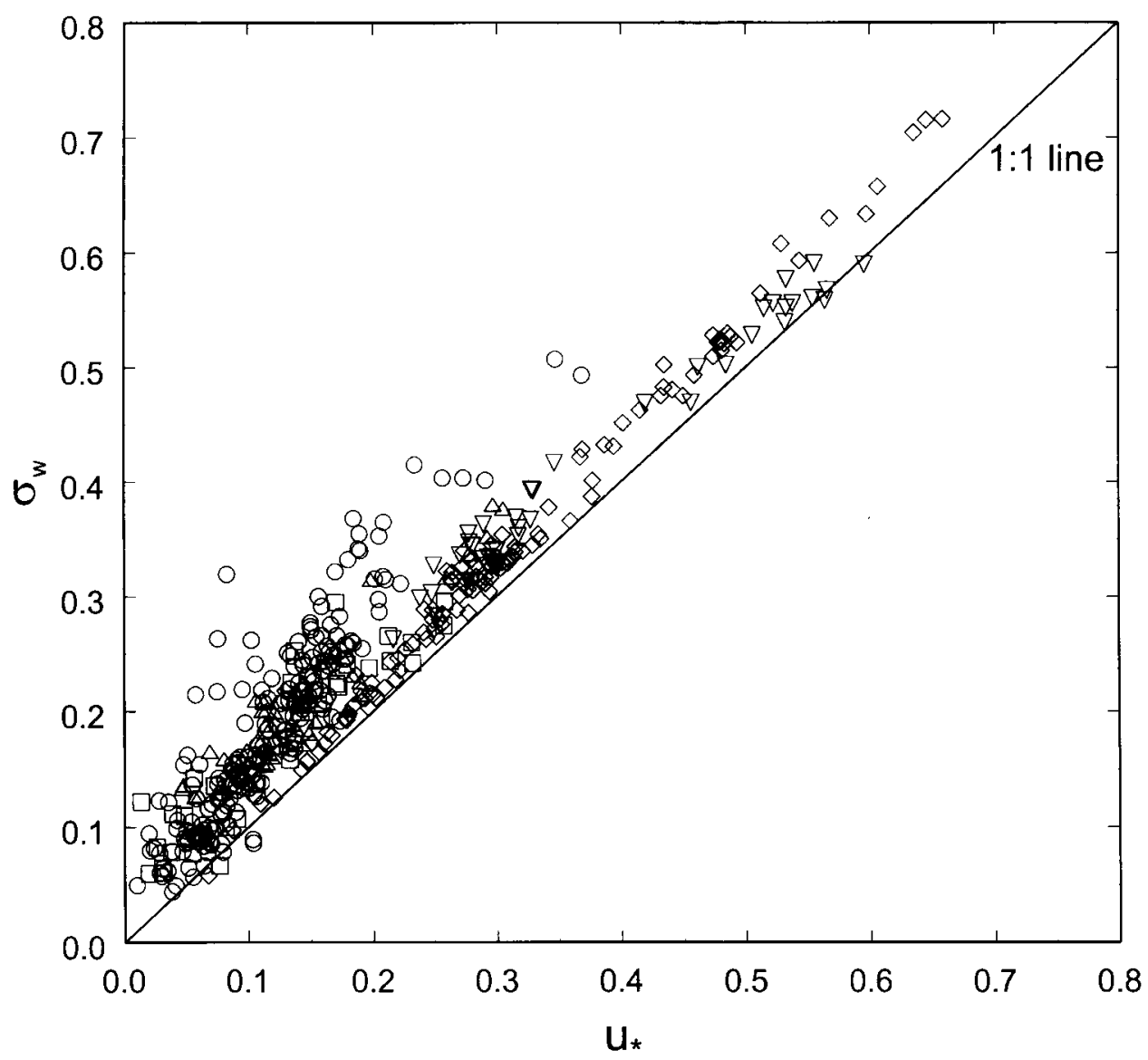

Figure 4. Standard deviation of the vertical velocity component $\sigma_{w}$ versus the friction velocity $u_{*}$ (symbols are the same as in Figure 1).

buoyancy effect and therefore the downward heat flux also decreases for very strong stability and nearly vanishes. In between those limits there exists a region of relatively strong downward heat flux. From the analyzed data this region was approximated to be $0.003 \lesssim z / L \lesssim 0.6$ (see Figure 6). Mahrt et al. (1998) found a height dependence for the maximum downward heat flux, which occurred at $z / L=0.6$ for data collected at $10-\mathrm{m}$ height and at $z / L=0.02$ for a height of $3 \mathrm{~m}$. Another study by Malhi (1995) revealed a maximum value for the heat flux at $z / L=0.2$. The results were based on data collected at $z=9 \mathrm{~m}$ in a nocturnal boundary layer in Niger, West Africa. In light of these results the dataset considered here also shows height dependence of the maximum downward heat flux. For the Davis 1995 data (diamonds in Figure 6), where the sensor height was lowest for all data considered in this study (see Table I), $-\left\langle w^{\prime} \theta^{\prime}\right\rangle_{\max }$ occurs approximately at $z / L=0.007$. In contrast to this, the Davis 1994 data (downward pointing triangles in Figure 6), collected at a height $1.54 \mathrm{~m}$, reveal a maximum downward 
ON MONIN-OBUKHOV SIMILARITY IN THE STABLE ATMOSPHERIC BOUNDARY LAYER 237

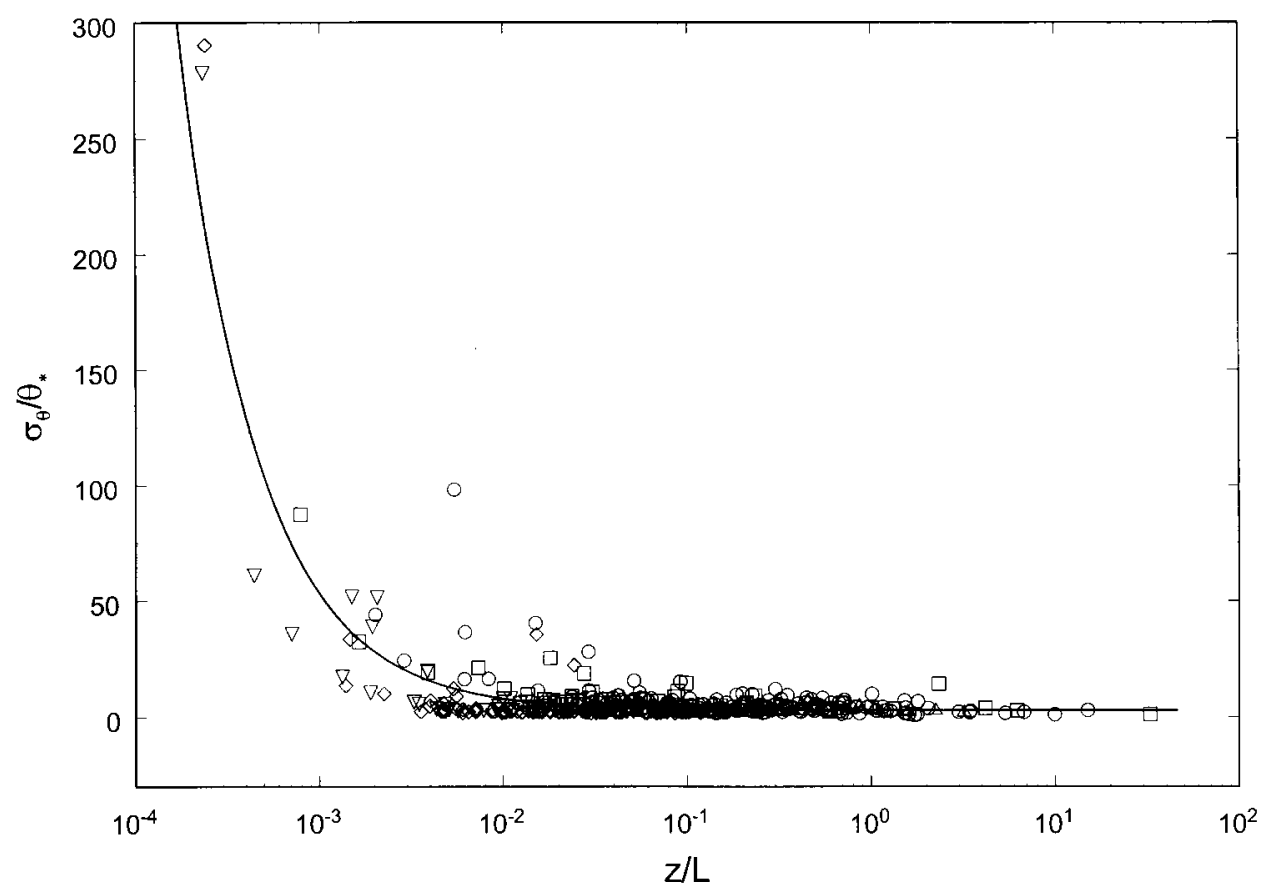

Figure 5a. Temperature standard deviation normalized with friction velocity $\theta_{*}$ (symbols are the same as in Figure 1).

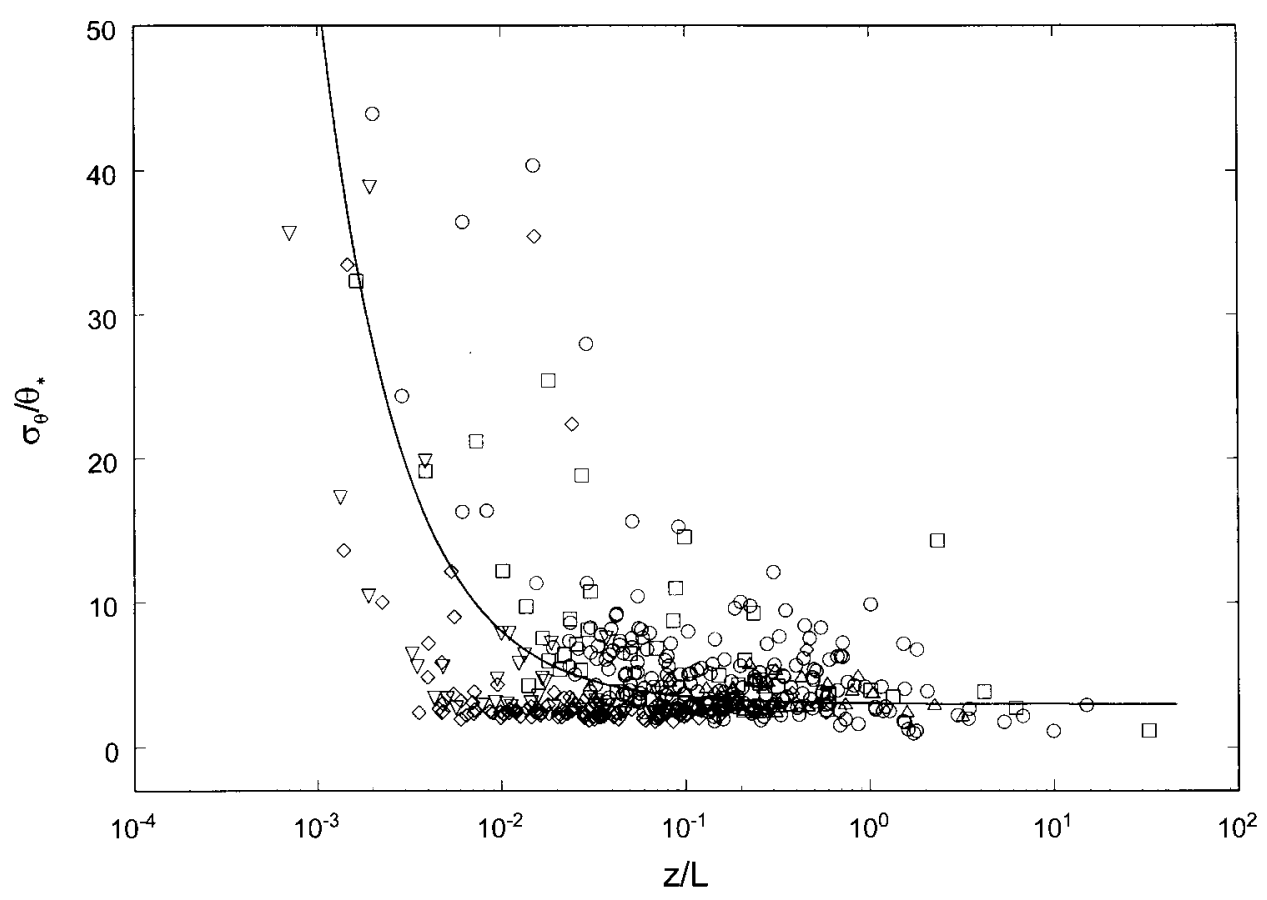

Figure 5b. Same as Figure 5a, but rescaled (symbols are the same as in Figure 1). 


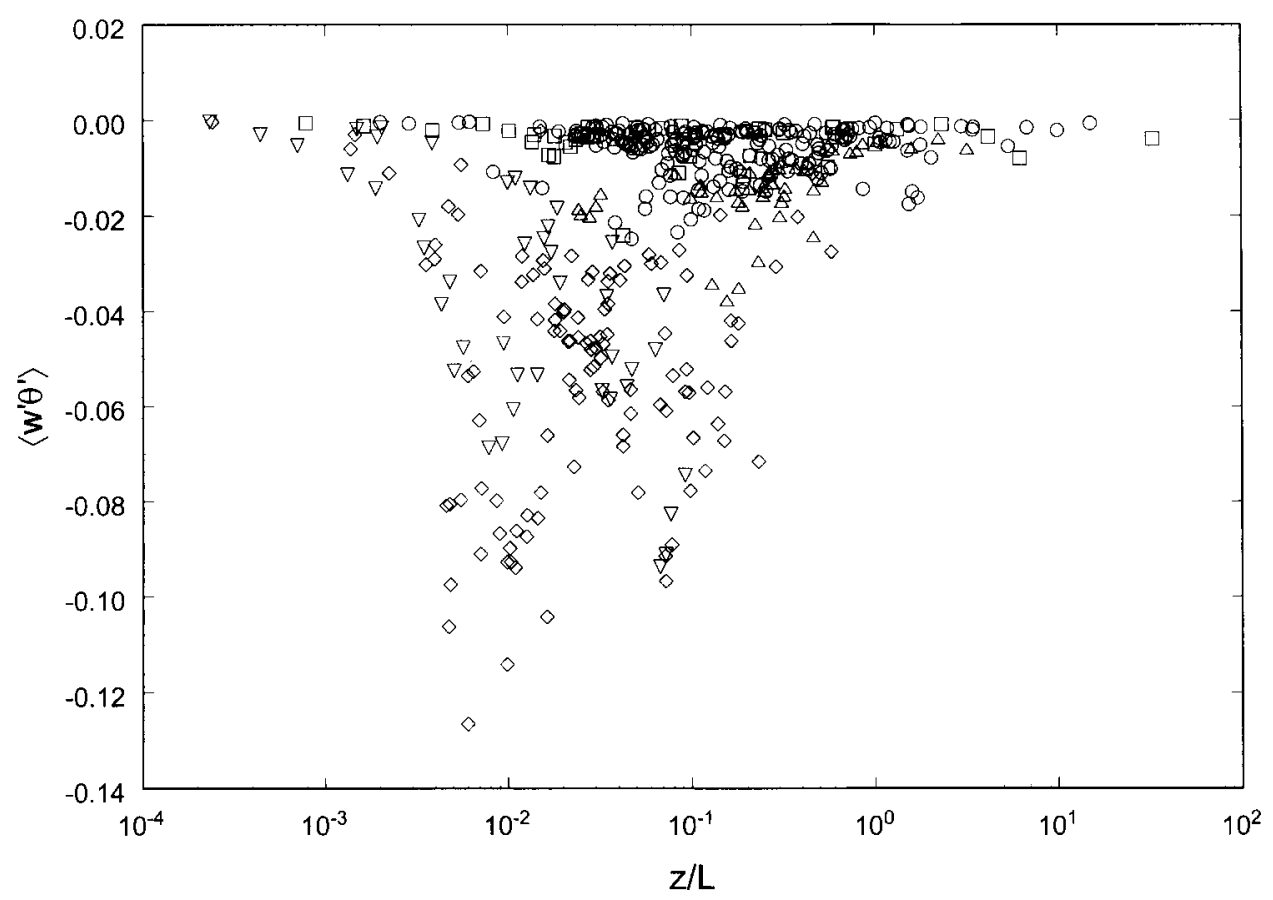

Figure 6. Heat flux $\left\langle w^{\prime} \theta^{\prime}\right\rangle$ as a function of $z / L$ (symbols are the same as in Figure 1).

heat flux at $z / L=0.07$, which agrees with the value obtained by Mahrt et al. (1998), but it should be noticed that a second maximum occurs at weaker stability $(z / L \approx 0.008)$. Upon removing the data taken during the field experiments in Davis 1994 and 1995 (low instrument heights), we obtain Figure 7 showing the remaining data collected at heights ranging from $1.63 \mathrm{~m}$ to $4.32 \mathrm{~m}$. The maximum downward heat flux occurs for $z / L=0.15$. This compares well with the result of Malhi (1995), even though the height difference between the two studies is significant. It is argued here that a height dependence for $-\left\langle w^{\prime} \theta^{\prime}\right\rangle_{\max }$ exists, and that the maximum value for downward heat flux does not have universal character.

The cross correlation between vertical velocity fluctuations and temperature fluctuations $r_{w \theta}\left(=\left\langle w^{\prime} \theta^{\prime}\right\rangle /\left(\sigma_{w} \sigma_{\theta}\right)\right)$ (see Figure 8) shows qualitatively the same behaviour as was seen for the heat flux. The maximum is shifted towards stronger stability and occurs at roughly $z / L=0.08$. The important fact that can be deduced from Figure 8 is the decrease of $r_{w \theta}$ for both limits, near neutral and strongly stable respectively, and a region of strong correlation in between those limits. In near-neutral stability the temperature fluctuations become very small and the temperature stratification almost vanishes, resulting in a weak cross correlation $r_{w \theta}$. The small values of $r_{w \theta}$ for very stable stratification need to be considered independently. In this case, where turbulence is very weak, large-scale motions (e.g., gravity waves) with low correlation between vertical velocity and temperature 


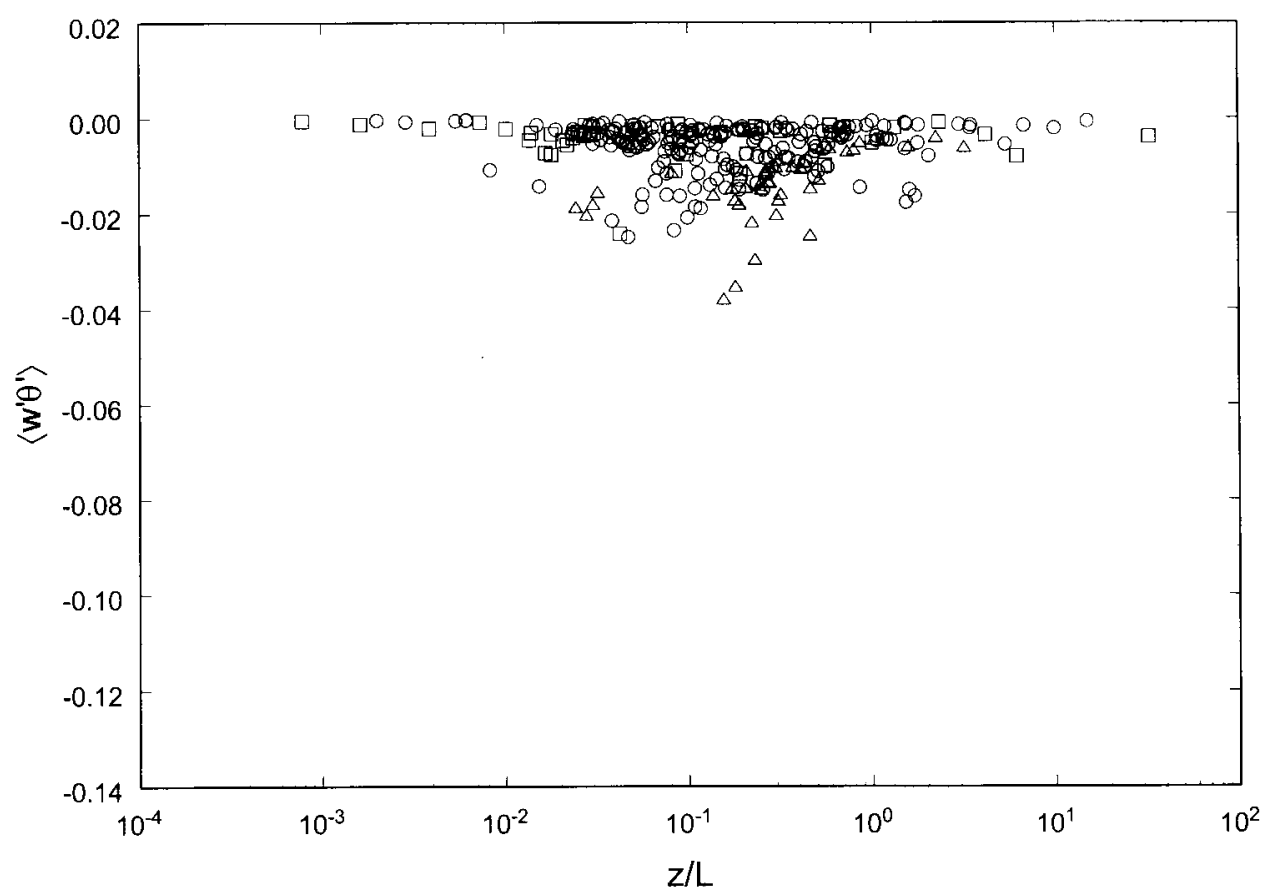

Figure 7. Same as Figure 6 but Davis 1994 and Davis 1995 data have been removed and only data for $z \geq 1.63 \mathrm{~m}$ is shown (symbols are the same as in Figure 1).

fluctuations predominate. The results obtained here can be viewed as an extension to the cross correlation $r_{w \theta}$ dependence on stability shown in Kaimal and Finnigan (1994, p. 20). From the arguments used above it should be clear that $-r_{w \theta}$ should become very small for the near-neutral and very stable limit. The cross correlation in between these limits does not take on a constant value for our data, as was suggested in Kaimal and Finnigan (1994).

\subsection{DisSiPATION OF TURBULENT KINETIC ENERGY AND TEMPERATURE VARIANCE}

In Figure 9 a typical plot of the third-order structure functions for longitudinal velocity and temperature is shown. They follow a scaling slope of 1 for a wide range of lag $r$, clearly indicating the existence of an inertial subrange. We apply an upper and lower limit to the lag in order to assure that the data for the calculations stem from the inertial subrange. The lower scale limit is based on Wyngaard's (1981) sonic-anemometer distortion criterion $\left(r=d_{s l}\right)$ and the upper limit is $r=$ $z / 2$, being a good approximation for the first $10 \mathrm{~m}$ of the atmospheric boundary layer (Kaimal, 1986). The third-order structure function for velocity has been used to delineate the inertial subrange, as it is considered to be a more stringent test of inertial subrange scaling than the second-order form (Anselmet et al., 1984; 


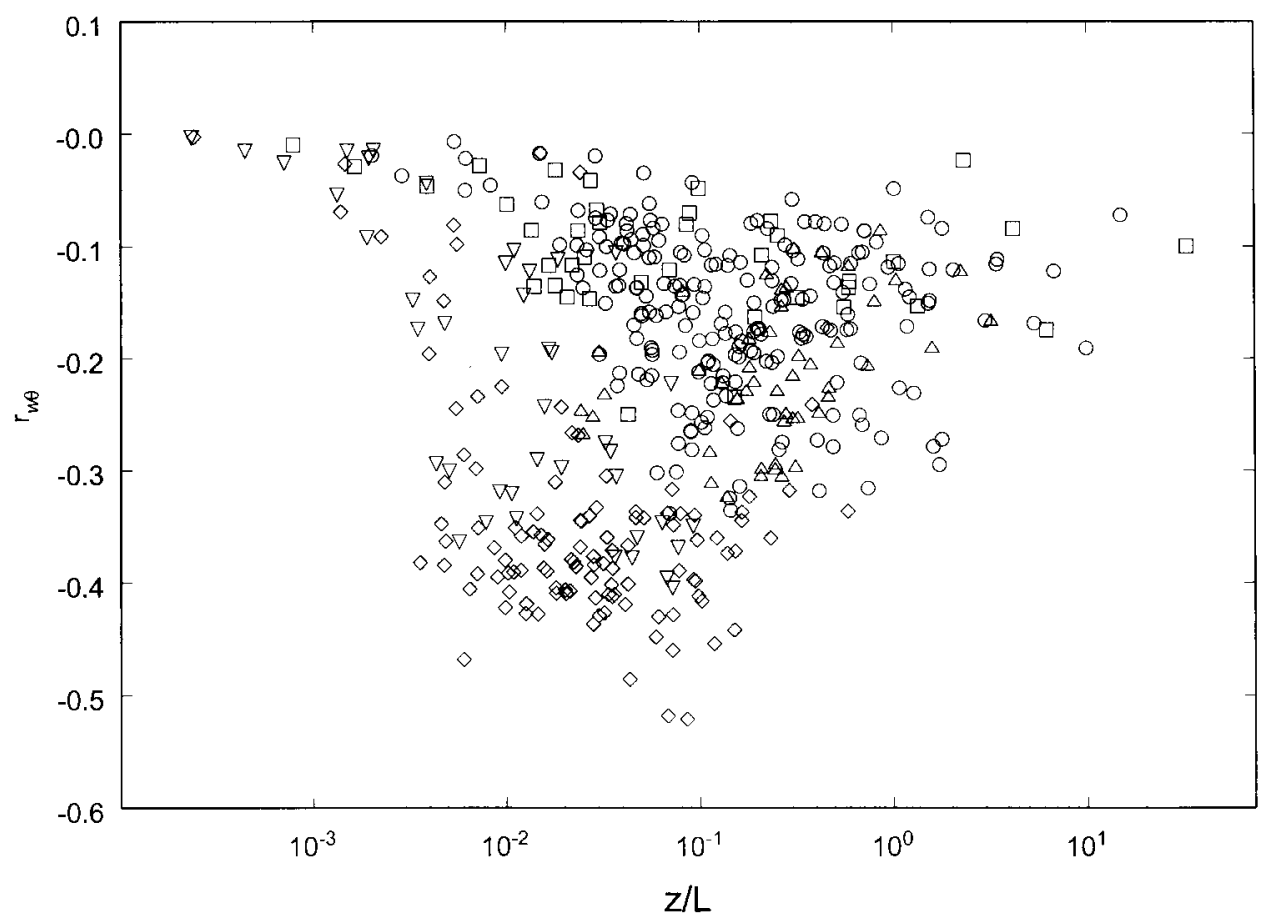

Figure 8. Cross correlation between vertical velocity and temperature fluctuations (symbols are the same as in Figure 1).

Kiely et al., 1996; Albertson et al., 1998). We use the third-order structure function forms of Kolmogorov's (1941) inertial subrange scaling to determine the average dissipation rates of turbulent kinetic energy and temperature variance.

First, we present the normalized average dissipation rate of TKE $\phi_{\epsilon}$, perhaps the most important but least discussed term in the normalized TKE equation (Frenzen and Vogel, 1992). Upon computing the average dissipation rate for all 446 runs according to (10) and (12) (the third-order structure function approach) and normalizing these values to $u_{*}^{3} / k z$, we obtain dissipation functions $\phi_{\epsilon}$ over a wide range of stability. This is shown in Figure 10. Both the second- and third-order structure function approaches have been used to compute the dissipation rate of TKE. We place more confidence on the results obtained with the third-order structure function, because the constant involved is exact $(-4 / 5)$. However, the resulting normalized dissipation rates show the same behaviour for both methods. For brevity, we do not present the results for the second-order structure function. For weak stability $\phi_{\epsilon}$ remains approximately constant, followed by a short transition period. Thereafter, $\phi_{\epsilon}$ increases strongly with increasing stability. The arguments made in the case of velocity and temperature fluctuations for the state of very stable stratification also apply for dissipation. It has been proposed that $z$ is no longer important for the stable limit (Monin and Yaglom, 1971). A generally accepted 

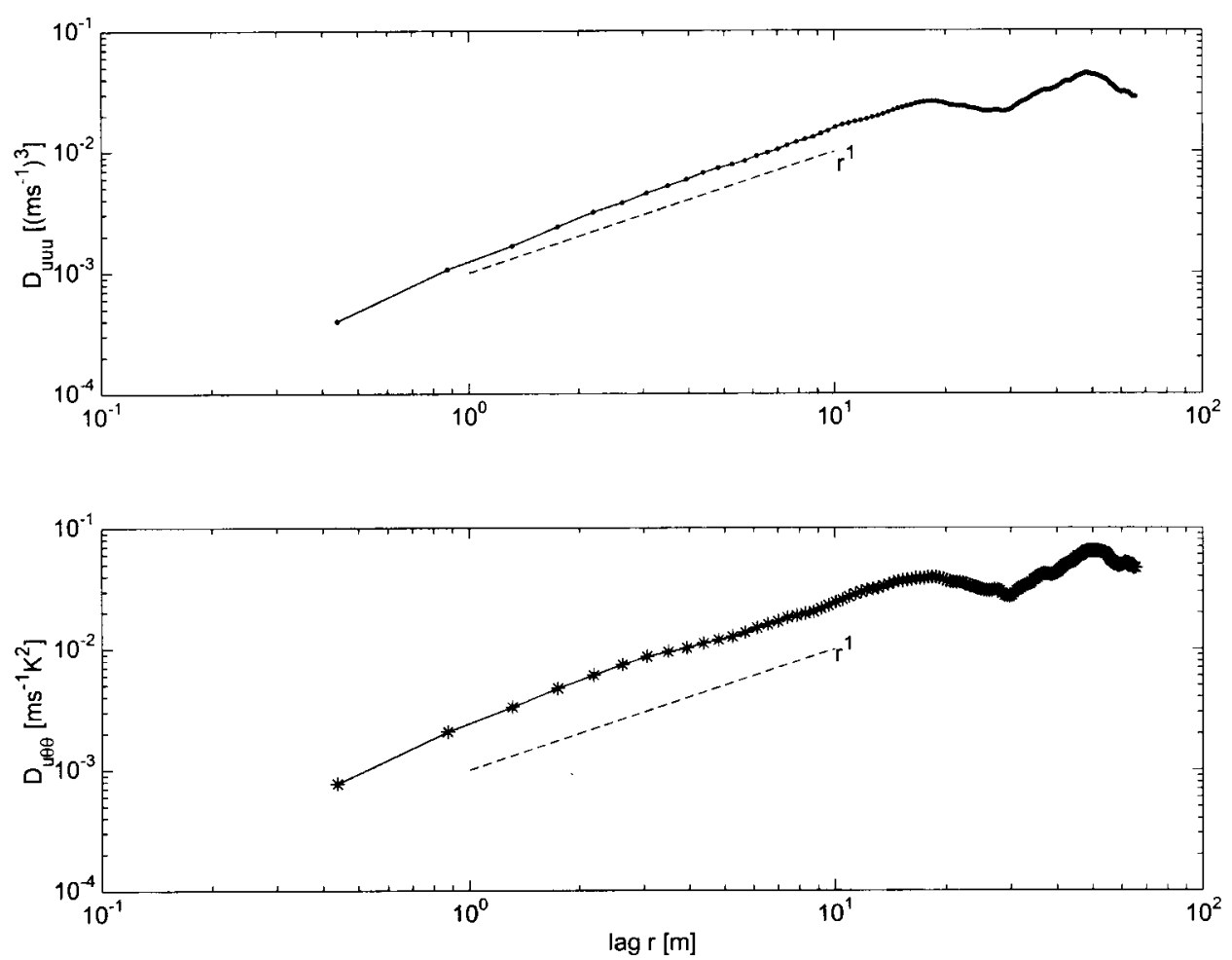

Figure 9. Third-order structure function for longitudinal velocity $D_{u и u}$ and mixed third-order structure function for temperature $D_{u \theta \theta}$.

prediction form for the normalized average dissipation rate is $\phi_{\epsilon}$ being proportional to the stability parameter:

$$
\phi_{\epsilon}=A+\gamma \frac{z}{L} .
$$

The additive constant $A$ is taken to be one in most cases, assuming production equals dissipation for neutral atmospheric conditions. Kaimal and Finnigan (1994) report the constant $\gamma=5$. An important point to be discussed here is that the data shown in Figure 10 do not suggest $\phi_{\epsilon}$ to be unity for neutral stability. As a consequence, production does not equal dissipation for neutral conditions. This was also shown by other researchers. Högström (1990b) finds the near-neutral dissipation rate of TKE to be approximately $25 \%$ larger than the production rate, and Frenzen and Vogel (1992) report dissipation to be about 15\% less than production. Both Högström, and Frenzen and Vogel, find that the Kansas data, analyzed by Wyngaard and Coté (1971), after re-evaluation, show that dissipation is less than production for near-neutral conditions. In a recent paper, Albertson et al. (1997) analyzed surface-layer data under neutral and unstable conditions. They found that in the neutral limit, the average dissipation rate of TKE was best described as being 


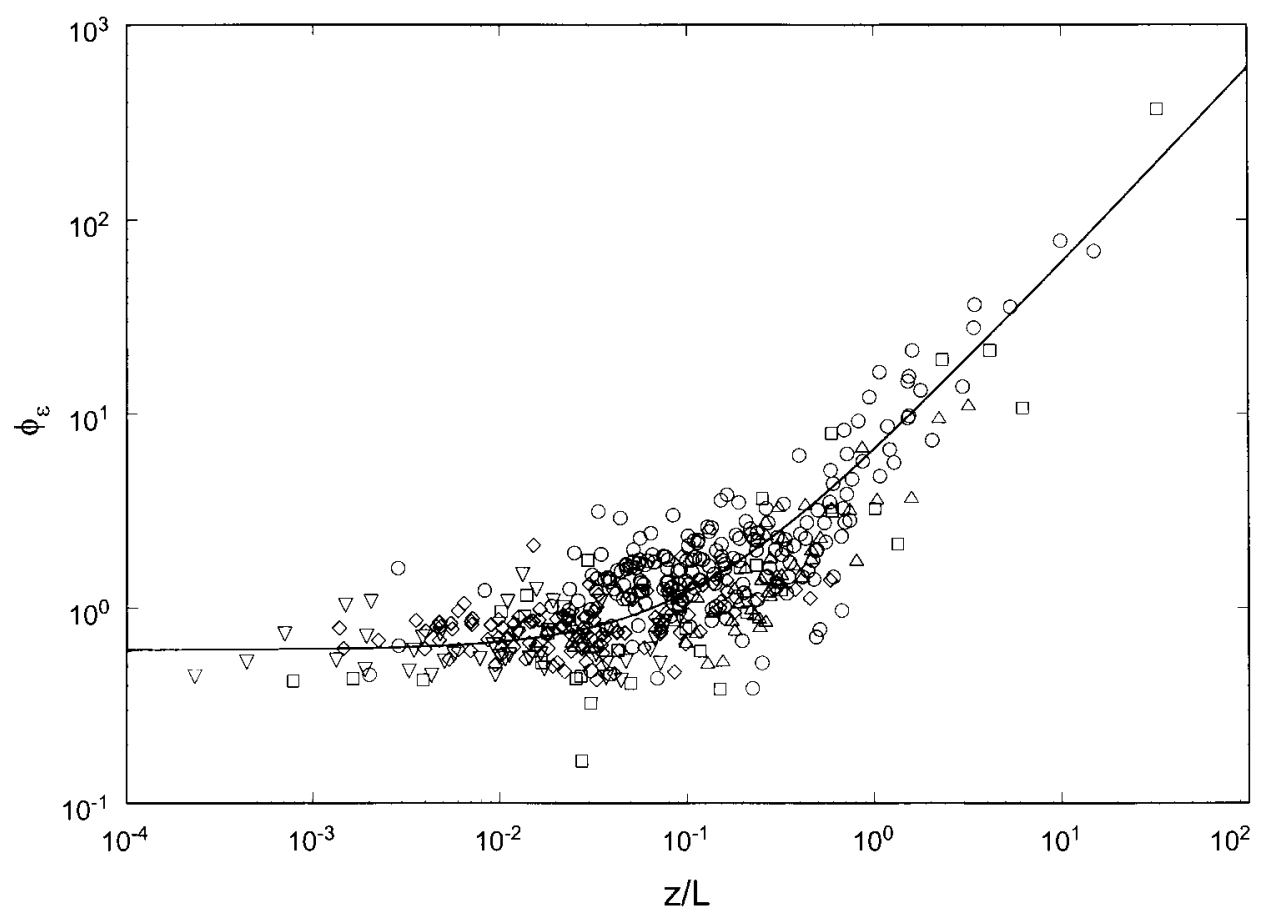

Figure 10. Normalized dissipation rate from third-order structure function (longitudinal velocity) (symbols are the same as in Figure 1).

constant $\phi_{\epsilon}=0.61$. This agrees very well with the data of this study, as can be seen from Figure 10. The continuous scaling function that best fits the data is

$$
\phi_{\epsilon}=0.61+5 \frac{z}{L}
$$

This function is plotted along with the data in Figure 10. The findings made are very satisfying in a number of ways. The expression found for the normalized average dissipation rate fits the observations over a stable range of more than five orders of magnitude. It also connects the unstable and stable sides of the stability regime. The result found by Albertson et al. (1997) that a constant value of 0.61 close to neutral best represents $\phi_{\epsilon}$ is a very good match to our data. Furthermore, our observations support an imbalance between production and dissipation near neutral, as was found by others. This imbalance has to be attributed to the flux divergence and pressure transport terms in order to close the TKE budget.

Consider the relative contribution of the two terms on the right hand side in Equation (16). For weak stability the first term on the right hand side dominates. The second term on the right hand side becomes important for more stable regimes. 
For the limit of very strong stability $A$ becomes very small compared to $\gamma(z / L)$ and can be neglected. Using the definition of $\phi_{\epsilon}$ we obtain

$$
\phi_{\epsilon}=\epsilon \frac{k z}{u_{*}^{3}}=\gamma(z / L)
$$

and

$$
\epsilon \frac{k L}{u_{*}^{3}}=\gamma,
$$

which demonstrates that the average dissipation rate of TKE obeys the concept of $z$-less stratification. Inserting the definition for $L$ (3), then $\epsilon=-\gamma \frac{g}{\langle\theta\rangle}\left\langle w^{\prime} \theta^{\prime}\right\rangle$, which states that dissipation equals a constant $\gamma$ times the buoyant destruction. For our data $\gamma=5$ provides a reasonably good fit.

We will now consider the dissipation of temperature variance. As for turbulent kinetic energy, the mixed third-order structure function approach has been chosen to obtain the dissipation rate. In order to calculate the average dissipation rate of temperature variance, $\epsilon_{\theta}$ with the second-order structure function, the average dissipation rate of TKE, $\epsilon$ must be known and a choice has to be made for the free constant that is involved. This adds uncertainty to the calculations and therefore we place more confidence on the results obtained with the mixed third-order approach. Figure 11 shows the resulting average dissipation rate of temperature variance, normalized by $k z u_{*} /\left\langle w^{\prime} \theta^{\prime}\right\rangle^{2}$. Interestingly, $\phi_{\epsilon \theta}$ is constant for almost the entire stability range covered by the observations. The large values for very weak stability $(\zeta<0.01)$ are due to the very small heat flux for near neutral conditions. Because $\phi_{\epsilon \theta}\left(=\epsilon_{\theta} k z u_{*} /\left\langle w^{\prime} \theta^{\prime}\right\rangle^{2}\right)$ is constant, the concept of $z$-less stratification does not apply in this case, since the $z$ dependence does not vanish for the very stable limit. The normalized dissipation rate of temperature variance does not increase with stability. The large scatter does not allow us much confidence in the average value for this constant behaviour; at best it can be said that $\phi_{\epsilon \theta}=1$ is a reasonable choice to describe the entire data set (except for near neutral conditions) (see Figure 11). The assumption $\phi_{h}-\phi_{\epsilon \theta}=0$ in the surface layer does not hold if $\phi_{h}=1+C(z / L)$ is applied, which is a commonly used scaling function for the normalized production. Several values for the constant $C$ have been suggested, e.g., $C=5$ (Dyer, 1974) or $C=7.8$ (Högström, 1988). However, because $\phi_{h}$ is a function that increases with stability, the imbalance between production and dissipation also increases for large $z / L$, given that the dissipation remains constant, as was found in the present work. There are two possible explanations for this discrepancy. One is that the transport term becomes important for the stably stratified boundary layer. If the stability function $\phi_{h}$ is linearly increasing with stability there has to be a third component to close the budget. The other explanation is that $\phi_{h}$ does in fact behave differently. Since $\phi_{h}$ has not been evaluated in this study no clear statement can be made about this puzzling behaviour. Howell and Sun (1999) calculated $\phi_{h}$ for a broad range 


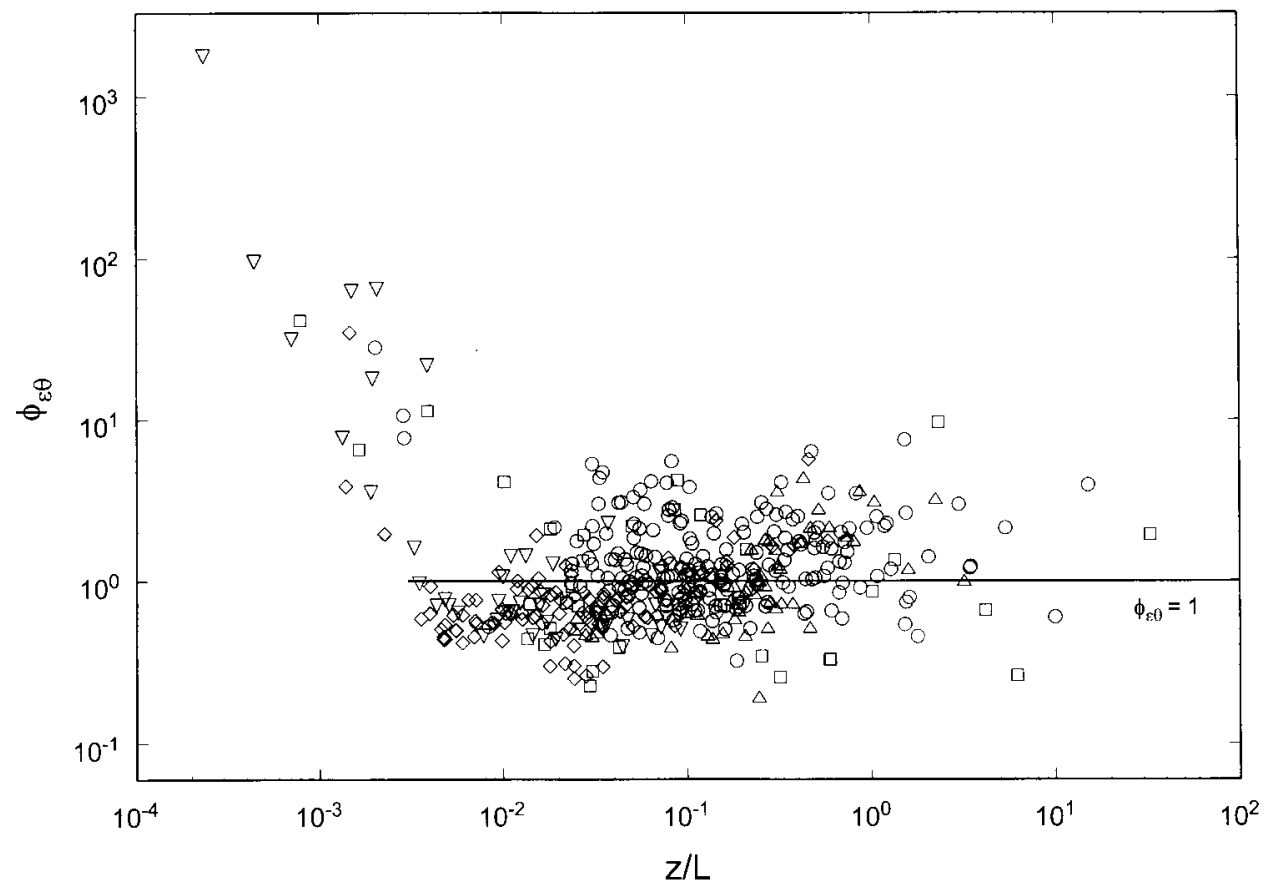

Figure 11. Normalized dissipation rate from mixed third-order structure function (temperature) (symbols are the same as in Figure 1).

of stability. They found that it increases with stability and levels off at around $z / L=0.5$. The constant value for $\phi_{h}$ for strong stability is difficult to identify due to large scatter (their Figure 7), but it seems to exceed the value found in the present study for $\phi_{\epsilon \theta}$. This suggests that the transport term becomes important in this stability regime. But further experimental investigations are certainly needed to confirm this conclusion.

\section{Conclusions}

Fast response data from measurements of the three wind velocity components and the air temperature in the surface layer using 3-dimensional sonic anemometers have been analyzed to gain insight into the structure of the stable ABL. Each individual run has been screened for the suitability of Taylor's frozen turbulence hypothesis to convert the data from the time to the space domain. Only runs with a turbulence intensity less than 0.5 have been selected, which resulted in a total number of 446 runs used for analysis. The applicability of Monin-Obukhov similarity theory under stable atmospheric conditions has been investigated. Moreover, we examined the validity of the concept of $z$-less stratification (Wyngaard and Coté, 1972) in the limit of very strong stability. The concept of $z$-less stratification 
implies that the turbulent eddies are no longer directly influenced by the surface as the atmosphere becomes very stable. It has been found that the non-dimensional standard deviations of the three velocity components $u, v$ and $w$ do not satisfy this criterion. For weak stability $(\zeta \lesssim 0.1)$ the normalized velocity standard deviations $\sigma_{\alpha} / u_{*}$, with $\alpha$ being $u, v$ and $w$, are constant. For increasing stability the $\sigma_{\alpha} / u_{*}$ increased strongly. In contrast to this result, the non-dimensional standard deviation of temperature $\sigma_{\theta} / \theta_{*}$ approaches a constant value for large $z / L$, being independent of $z$ in the very stable regime.

Our suggested fit for the normalized velocity standard deviations (14) captures the quick transition of these variables from being constant in the weakly stable region to a form that increases with $z / L$ for very strong stability. For the nondimensional standard deviation of temperature an expression (15) has been found that represents the data over the entire stable regime.

The average dissipation rate, $\epsilon$ of turbulent kinetic energy and the average dissipation rate of temperature variance, $\epsilon_{\theta}$ have been calculated using the secondand third-order structure function approaches. The third-order approach must be viewed as being superior to that using the second-order structure function, since no free constants are involved. Therefore the conclusions drawn were based on results obtained with the third-order approach. It has been found that the non-dimensional average dissipation rate of TKE, $\phi_{\epsilon}$ does become $z$-less for the very stable limit. On the other hand, the non-dimensional average dissipation rate of temperature variance, $\phi_{\epsilon \theta}$ does not support the concept of $z$-less stratification.

The extensive data set used here, covering a stability range of about five orders of magnitude of the stability parameter $\zeta$, revealed that the concept of $z$-less stratification is not valid in general.

It has also been found that there is an imbalance between production and dissipation of TKE for the neutral limit. For the TKE budget to reach closure, the transport terms have to be taken into account, a result which is in agreement with Albertson et al. (1997).

\section{Acknowledgments}

The authors wish to thank our colleagues, Charles Meneveau, Elizabeth Jacobs, Pamela Rawe and Joe Arreola from Johns Hopkins University, Roger Shaw and Mike Mata from U. C. Davis and William Eichinger, Ahmed Lachhab and Zhiming Chen from University of Iowa for discussions and for their assistance in the field. We also thank Gabriel Katul from Duke University and Jay Ham from Kansas State University for the loan of equipment. We are grateful to Larry Mahrt and an anonymous reviewer for their valuable comments that helped to improve the manuscript. The authors gratefully acknowledge funding from NSF-ATM-9726270 and USDA-98-35107-6346. 


\section{References}

Albertson, J. D., Katul, G. G., Parlange, M. B., and Eichinger, W. E.: 1998, 'Spectral Scaling of Static Pressure Fluctuations in the Atmospheric Surface Layer: The Interaction between Large and Small Scales', Phys. Fluids 10, 1725-1732.

Albertson, J. D., Parlange, M. B., Kiely, G., and Eichinger, W. E.: 1997, 'The Average Dissipation Rate of Turbulent Kinetic Energy in the Neutral and Unstable Atmospheric Surface Layer', $J$. Geophys. Res. 102, 13423-13432.

Anselmet, F., Gagne, Y., Hopfinger, E. J., and Antonia, R. A.: 1984, 'High-Order Velocity Structure Functions in Turbulent Shear Flows', J. Fluid Mech. 140, 63-89.

Beljaars, A. C. M. and Holtslag, A. A. M.: 1991, 'Flux Parameterization over Land Surfaces for Atmospheric Models', J. Appl. Meteorol. 30, 327-341.

Brutsaert, W.: 1992, 'Stability Correction Functions for the Mean Wind Speed and Temperature in the Unstable Surface Layer', Geophys. Res. Lett. 19, 469-472.

Brutsaert, W.: 1999, 'Aspects of Bulk Atmospheric Boundary Layer Similarity under FreeConvective Conditions', Rev. Geophys. 37, 439-451.

Businger, J. A., Wyngaard, J. C., Izumi, Y., and Bradley, E. F.: 1971, 'Flux-Profile Relationships in the Atmospheric Surface Layer', J. Atmos. Sci. 28, 181-189.

Caughey, S. J., Wyngaard, J. C., and Kaimal, J. C.: 1979, 'Turbulence in the Evolving Stable Boundary Layer', J. Atmos. Sci. 36, 1041-1052.

Champagne, F. H., Friehe, C. A., and Larue, J. C.: 1977, 'Flux Measurements, Flux Estimation Techniques, and Fine-Scale Turbulence Measurements in the Unstable Surface Layer over Land', J. Atmos. Sci. 34, 515-530.

Chu, C. R, Parlange, M. B., Katul, G. G., and Albertson, J. D.: 1996, 'Probability Density Functions of Turbulent Velocity and Temperature in the Atmospheric Surface Layer', Water Resour. Res. 32, 1681-1688.

De Bruin, H. A. R., Kohsiek, W., and van den Hurk, J. J. M.: 1993, 'A Verification of Some Methods to Determine the Fluxes of Momentum, Sensible Heat, and Water Vapour Using Standard Deviation and Structure Parameter of Scalar Meteorological Quantities', Boundary-Layer Meteorol. 63, 231-257.

Dias, N. L. and Brutsaert, W.: 1996, 'Similarity of Scalars under Stable Stratification', BoundaryLayer Meteorol. 80, 355-373.

Dias, N. L., Brutsaert, W., and Wesley, M. L.: 1995, 'z-Less Stratification under Stable Conditions', Boundary-Layer Meteorol. 75, 175-187.

Dyer, A. J.: 1974, 'A Review of Flux-Profile Relationships', Boundary-Layer Meteorol. 7, 363-372.

Dyer, A. J. and Hicks, B. B.: 1970, 'Flux-Gradient Relationships in the Constant Flux Layer', Quart. J. Roy. Meteorol. Soc. 96, 715-721.

Finnigan, J.: 1999, 'A Note on Wave-Turbulence Interaction and the Possibility of Scaling the Very Stable Boundary Layer', Boundary-Layer Meteorol. 90, 529-539.

Forrer, J. and Rotach, M. W.: 1997, 'On the Turbulence Structure in the Stable Boundary Layer over the Greenland Ice Sheet', Boundary-Layer Meteorol. 85, 111-136.

Frenzen, P. and Vogel, C. A.: 1992, 'The Turbulent Kinetic Energy Budget in the Atmospheric Surface Layer: A Review and an Experimental Reexamination in the Field', Boundary-Layer Meteorol. 60, 49-76.

Högström, U.: 1988, 'Non-Dimensional Wind and Temperature Profiles in the Atmospheric Surface Layer: A Re-Evaluation', Boundary-Layer Meteorol. 42, 55-78.

Högström, U.: 1990a, 'Analysis of Turbulence Structure in the Surface Layer in Near-Neutral Conditions', in Proceeding of the Ninth Symposium on Turbulence and Diffusion, Røskilde, Denmark, April 30-May 3, 1990, Amer. Meteorol. Soc., pp. 235-236.

Högström, U.: 1990b, 'Analysis of Turbulence Structures in the Surface Layer with a Modified Similarity Formulation for Near Neutral Conditions’, J. Atmos. Sci. 47, 1949-1972. 
Howell, J. F. and Sun, J.: 1999, 'Surface-Layer Fluxes in Stable Conditions', Boundary-Layer Meteorol. 90, 495-520.

Hsieh C. I. and Katul, G. G.: 1997, 'Dissipation Methods, Taylor's Hypothesis, and Stability Correction Functions in the Atmospheric Surface Layer', J. Geophys. Res. 102, 16391-16405.

Kader, B. A. and Yaglom, A. M.: 1990, 'Mean Fields and Fluctuation Moments in Unstably Stratified Turbulent Boundary Layers', J. Fluid Mech. 212, 637-662.

Kaimal, J. C.: 1986, 'Flux and Profile Measurements from Towers in the Boundary Layer', in D. H. Lenschow (ed.), Probing the Atmospheric Boundary Layer, Amer. Meteorol. Soc., pp. 19-28.

Kaimal, J. C. and Finnigan, J. J.: 1994, Atmospheric Boundary Layer Flows: Their Structure and Measurement, Oxford University Press, $289 \mathrm{pp}$.

Kaimal, J. C., Wyngaard, J. C., Haugen, D. A., Coté, O. R., Izumi, Y., Caughey, S. J., and Readings, C. J.: 1976, 'Turbulence Structure in the Convective Boundary Layer', J. Atmos. Sci. 33, 21522169.

Katul, G. G., Hsieh, C. I., and Sigmon, J.: 1997, 'Energy-Inertial Scale Interactions for Velocity and Temperature in the Unstable Atmospheric Surface Layer', Boundary-Layer Meteorol. 82, 49-80.

Kiely, G., Albertson, J. D., Parlange, M. B., and Eichinger, W. E.: 1996, 'Convective Scaling of the Average Dissipation Rate of Temperature Variance in the Atmospheric Surface Layer', Boundary-Layer Meteorol. 77, 267-284.

Kolmogorov, A. N.: 1941, 'The Local Structure of Turbulence in Incompressible Viscous Fluid for Very Large Reynolds Numbers', Dokl. Akad. Nauk. SSSR 30, 301-303.

Kondo, J., Kanechika, O., and Yasuda, N.: 1978, 'Heat and Momentum Under Strong Stability in the Atmospheric Surface Layer', J. Atmos. Sci. 35, 1012-1021.

Mahrt, L.: 1985, 'Vertical Structure and Turbulence in the Very Stable Boundary Layer', J. Atmos. Sci. 42, 2333-2349.

Mahrt, L.: 1998, 'Stratified Atmospheric Boundary Layers and Breakdown of Models', Theoret. Comput. Fluid Dyn. 11, 263-279.

Mahrt, L.: 1999, 'Stratified Atmospheric Boundary Layers', Boundary-Layer Meteorol. 90, 375-396.

Mahrt, L., Sun, J., Blumen, W., Delany, T., and Oncley, S.: 1998, 'Nocturnal Boundary-Layer Regimes', Boundary-Layer Meteorol. 88, 255-278.

Malhi, Y. S.: 1995, 'The Significance of the Dual Solutions for Heat Fluxes Measured by the Temperature Fluctuation Method in Stable Conditions', Boundary-Layer Meteorol. 74, 389-396.

Merry, M. and Panofsky, H. A.: 1976, 'Statistics of Vertical Motion over Land and Water', Quart. J. Roy. Meteorol. Soc. 102, 255-260.

Monin, A. S. and Obukhov, A. M..: 1954, 'Basic Laws of Turbulent Mixing in the Ground Layer of the Atmosphere', Trans. Geophys. Inst. Akad. Nauk. USSR 151, 163-187.

Monin, A. S. and Yaglom, A. M.: 1971, Statistical Fluid Mechanics: Mechanics of Turbulence, Vol. 1, MIT Press, Cambridge, MA, 769 pp.

Monin, A. S. and Yaglom, A. M.: 1975, Statistical Fluid Mechanics: Mechanics of Turbulence, Vol. 2, MIT Press, Cambridge, MA, 874 pp.

Nieuwstadt, F. T. M.: 1984a, 'The Turbulent Structure of the Stable, Nocturnal Boundary Layer', J. Atmos. Sci. 41, 2202-2216.

Nieuwstadt, F. T. M.: 1984b, 'Some Aspects of the Turbulent Stable Boundary Layer', BoundaryLayer Meteorol. 30, 31-55.

Panofsky, H. A. and Dutton, J. A.: 1984, Atmospheric Turbulence: Models and Methods for Engineering Applications, Wiley and Sons, $397 \mathrm{pp}$.

Parlange, M. B. and Katul, G. G.: 1995, 'Watershed Scale Shear Stress from Tethersonde Wind Profile Measurements under Near Neutral and Unstable Atmospheric Stability', Water Resour. Res. 31, 961-968.

Parlange, M. B., Albertson, J. D., Eichinger, W. E., Cahill, A. T., Jackson, T. J., Kiely, G., and Katul, G. G.: 1999, 'Evaporation: Use of Fast-Response Turbulence Sensors, Raman Lidar, and 
Passive Microwave Remote Sensing', in M. B. Parlange and J. W. Hopmans (eds.), Vadose Zone Hydrology: Cutting across Disciplines, Oxford University Press, 454 pp.

Parlange, M. B., Eichinger, W. E., and Albertson, J. D.: 1995, 'Regional Scale Evaporation and the Atmospheric Boundary Layer', Rev. Geophys. 33, 99-124.

Peltier, L. J., Wyngaard, J. C., Khanna, and Brasseur, J. G.: 1996, 'Spectra in the Unstable Surface Layer', J. Atmos. Sci. 53, 49-61.

Porté-Agel, F., Meneveau, C., and Parlange, M. B.: 1998, 'Some Basic Properties of the Surrogate Subgrid-Scale Heat Flux in the Atmospheric Boundary Layer', Boundary-Layer Meteorol. 88, 425-444.

Porté-Agel, F., Parlange, M. B., Meneveau, C., Eichinger, W. E., and Pahlow, M.: 2000, 'Subgridscale Dissipation in the Atmospheric Surface Layer: Effects of Stability and Filter Dimension', J. Hydrometeorol. 1, 75-87.

Porté-Agel, F., Parlange, M. B., Meneveau, C., and Eichinger, W. E.: 2001, 'A Priori Field Study of the Subgrid-Scale Heat Fluxes and Dissipation in the Atmospheric Surface Layer', J. Atmos. Sci., in press.

Smedman, A.: 1988, 'Observations of a Multi-Level Turbulence Structure in a Very Stable Boundary Layer', Boundary-Layer Meteorol. 44, 231-253.

Sorbjan, Z.: 1986, 'On Similarity Theory in the Atmospheric Boundary Layer', Boundary-Layer Meteorol. 34, 377-397.

Stull, R. B.: 1988, 'An Introduction to Boundary Layer Meteorology, Kluwer Academic Publishers, Dordrecht, $670 \mathrm{pp}$.

Takeuchi, K.: 1961, 'On the Structure of the Turbulent Field in the Surface Boundary Layer', J. Meteorol. Soc. Jpn. 39, 346-364.

Taylor, G. I.: 1938, 'The Spectrum of Turbulence', Proc. Roy. Soc. London A164, 476-490.

Tong, C. N., Wyngaard, J. C., and Brasseur, J. G.: 1999, 'Experimental Study of the Subgrid-Scale Stresses in the Atmospheric Surface Layer', J. Atmos. Sci. 56, 2277-2292.

Tong, C. N., Wyngaard, J. C., Khanna, S., and Brasseur, J. G.: 1998, 'Resolvable and Subgrid-Scale Measurement in the Atmospheric Surface Layer: Technique and Issues', J. Atmos. Sci. 55, 31143126.

von Karman, T. and Howarth, L.: 1938, 'On the Statistical Theory of Isotropic Turbulence', Proc. Roy. Soc. London A164, 192-215.

Wang, J. and Mitsuta, Y.: 1991, 'Turbulence Structure and Transfer Characteristics in the Surface Layer of the HEIFE Gobi Area', J. Meteorol. Soc. Japan 69, 587-593.

Wyngaard, J. C.: 1981, 'Cup, Propeller, Vane, and Sonic Anemometers in Turbulence Research', Annu. Rev. Fluid Mech. 13, 399-423.

Wyngaard, J. C. and Clifford, S. F.: 1977, 'Taylor's Hypothesis and High-Frequency Turbulence Spectra', J. Atmos. Sci. 34, 922-929.

Wyngaard, J. C. and Coté, O. R.: 1971, 'The Budgets of Turbulent Kinetic Energy and Temperature Variance in the Atmospheric Surface Layer', J. Atmos. Sci. 28, 190-201.

Wyngaard, J. C. and Coté, O. R.: 1972, 'Cospectral Similarity in the Atmospheric Surface Layer', Quart. J. Roy. Meteorol. Soc. 98, 590-603.

Wyngaard, J. C., Coté, O. R., and Izumi, Y.: 1971, 'Local Free Convection, Similarity, and the Budgets of Shear Stress and Heat Flux', J. Atmos. Sci. 28, 1171-1182.

Yaglom, A. M.: 1949, 'Homogeneous and Isotropic Turbulence in a Viscous Compressible Fluid', Izv. Akad. Nauk. SSSR, Ser. Geogr. i Geofiz. 12, 501-522. 